\title{
Expression patterns of the Siah genes in postnatal mouse skin
}

\author{
Tverye E. A. ${ }^{1}$, Rudyak S.G. ${ }^{2}$, Shinin V. ${ }^{1}{ }^{1}$, Panteleyev A.A. ${ }^{1}$ \\ 1- National Research Center «Kurchatov Institute», Moscow, Russia \\ 2- N.M. Emanuel Institute of Biochemical Physics RAS, Moscow, Russia
}

NATIONAL

\section{Introduction:}

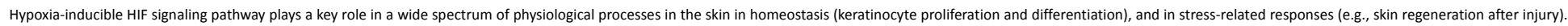

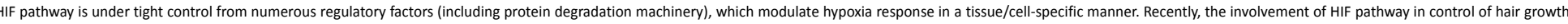

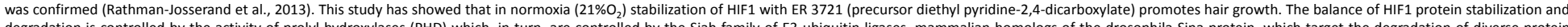

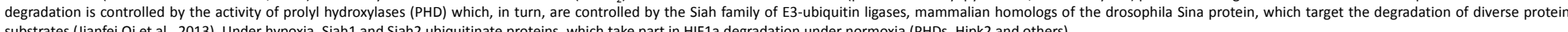
substrates (Jianfei Qi et al., 2013). Under hypoxia, Siah1 and Siah2 ubiquitinate proteins, which take part in HIF1a degradation under normoxia (PHDs, Hipk2 and others).

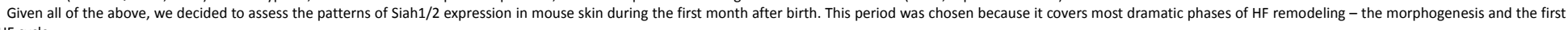
HF cycle.

Materials and methods:

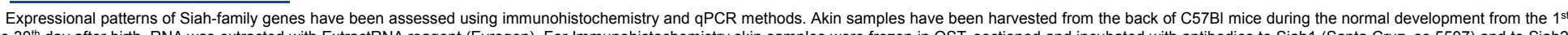

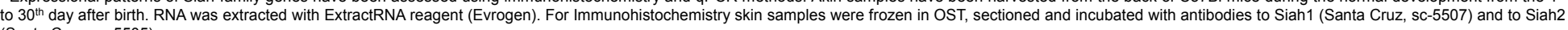
(Santa Cruz, sc-5505)

Results 1:

Siah 1 expression is primarily associated with epithelial skin compartment (epidermis and HF) and is hair cycle-dependent

Immunohistochemistry shows that Siah1 positivity localizes to epithelial parts of the skin (epidermis and hair follicles). After birth during the normal development protein changes its expression pattern. These changes can be associated with epithelial development and hair follicle cycle (Fig.1).

In the epidermis, Siah1 protein localizes to all basal and suprabasal layers from he $1^{\text {st }}$ day after birth to $5^{\text {th }}$ day. Then, Siah1 is detected in suprabasal layers, but at $2^{\text {th }}$ day of postnatal development the protein localizes exclusively to granular layer where it stays until $17-19^{\text {th }}$ days after birth. Form this time point and up to the $30^{\text {th }}$ ay, the Siah1 is detected in suprabasal epidermal layers.

In hair follicle, Siah1 is detected in inner root sheath (IRS) during first month of mice life. Protein also localizes in outer root sheath (ORS) in late stages of hair follicle morphogenesis. During stages 7 and 8 of HF morphogenesis Siah1 is seen in lower portion of both root sheaths. In early catagen, Siaht is expressed in IRS,

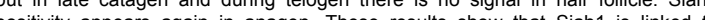
positivy appears again in angen. These results show that Sian is linked to keratinocyte differentiation in hair follcle duning normal development and hair cycle.

$$
\begin{array}{lccccccc}
\text { Stage } 4 & \text { Stage 6 } & \text { Stage 7 } & \text { Stage 8 } & \text { Catagen I } & \text { Catagen VII } & \text { Telogen } & \text { Anagen IV } \\
0 \mathrm{ppp} & 3 \mathrm{pp} & 5-9 \mathrm{pp} & 12 \mathrm{pp} & 15 \mathrm{pp} & 17 \mathrm{pp} & 19-22 \mathrm{pp} & 30 \mathrm{pp}
\end{array}
$$

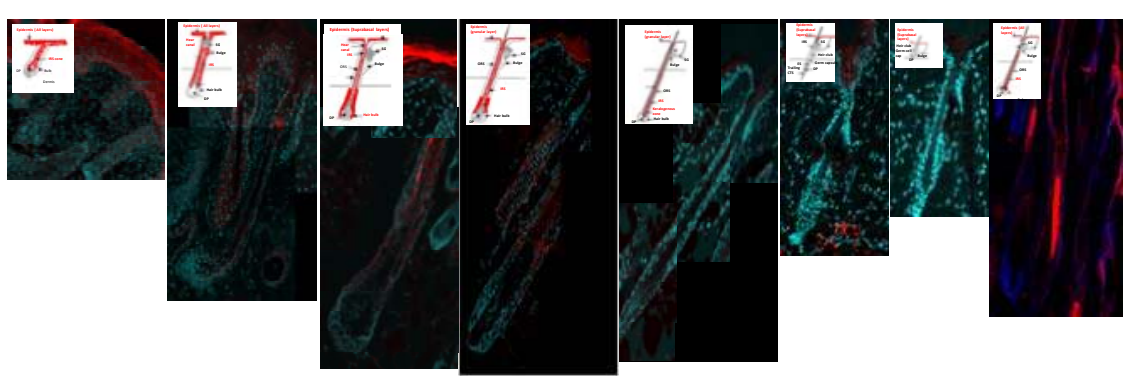

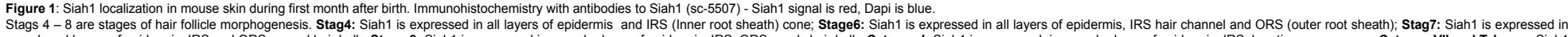

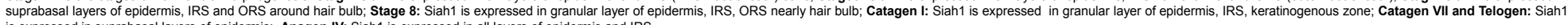
is expressed in suprabasal layers of epldermis, Anagen IV: S: $S$ is 8 : ST

Schemes are adopted from articles R. Paus et al., 1999 and S. Müller-Röver et al., 200

Results 2:

Siah2 expression is detected in mesenchymal skin compartment (dermis, dermal papilla and dermal sheath) and is hair cycle-dependent

Siah2 has primarily dermal localization. During the first month of mice life protein are seen in dermis. It also is detected in dermal sheath and in dermal papilla. Interestingly, in dermal papilla protein is expressed during stages of $\mathrm{HF}$ active growth and degradation (morphogenesis, anagen and catagen) (Fig.2).

Siah2 is also detected in epidermis during days $7-12$ of postnatal development.

The signal is seen exclusively in suprabasal epidermal layers.

These results suggest that Siah2 takes part in the processes associated with remodeling of the dermis.

Figure 2: Siah2 localization in mouse skin during first month after birth. Immunohistochemistry with Figure 2: Siah2 localization in mouse skin during first mon
antibodies to Siah2 (sc-5505) - Siah2 is in Red, Dapi is blue.

Siah2 expression during hair follicle morphogenesis. Stage 4: Siah2 is expressed in the dermis an in DP (dermal papilla); Stage 6: Siah2 is expressed in the dermis and DP; Stage 7: Siah2 expressed in suprabasal layers of the epldermis, in the dermis and in dermal sheath; Stage Siah2 is expressed in granular layer of the epidermis, in the dermis and dermal sheath; Catagen

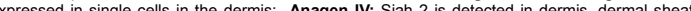
and DP. Other Abbreviations: DP. dermal papilla, SG-sebaceous gland, ES- epithelial strand, CTS- connective tissue sheath, IRS - Inner root sheath, ORS - outer root sheath

In schemes* Siah2 localization is shown in Red.
Schemes are adopted from articles R. Paus et al., 1999 and S. Müller-Röver et al., 2001

Results 3: $\quad$ Expression of Siah family genes in mice skin during firs month after birth

Expression of Siah-family genes (qPCR) in the full-skin samples during first 30 days of postnatal mouse development.

A: two peaks of Siah 1 expression are seen at days 12 and 15 after birth. This time-interval corresponds to the end of morphogenesis and beginning of the first catagen.

B: Siah2 mRNA level in whole-skin samples (qPCR). The high level of expression is seen at days 5,12 and 30 after birth. The $5^{\text {th }}$ and 30 th days are periods of active hair grow while the $12^{\text {th }}$ day demarcates transition from morphogenesis and catagen

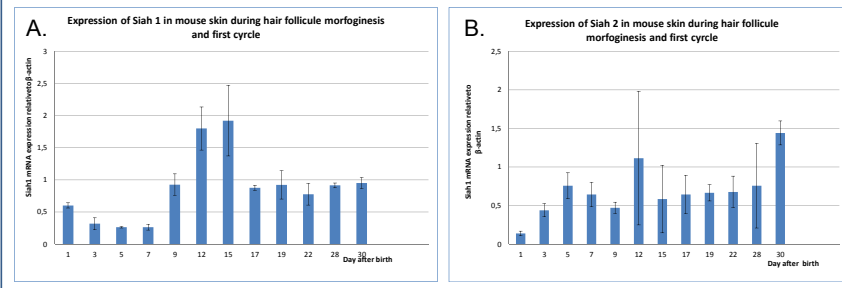

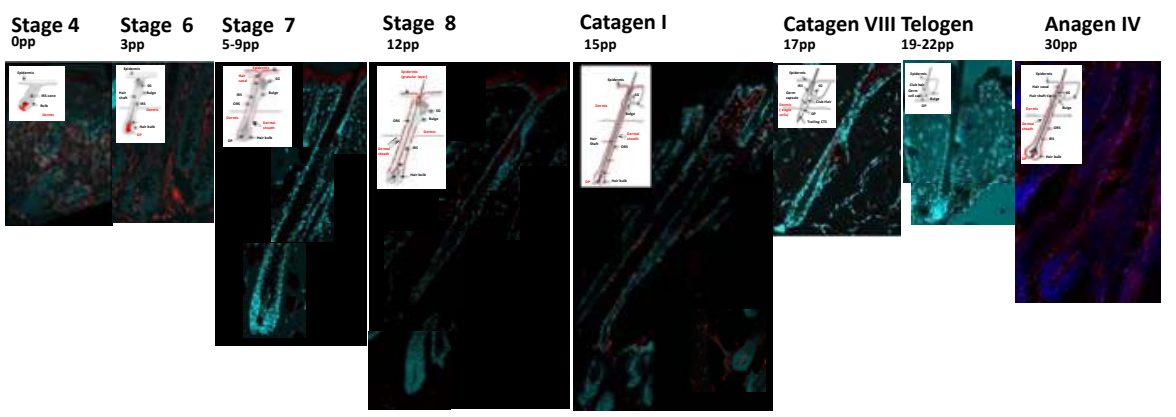

\section{Conclusions:}

Siah1 positivity is primarily associated with epithelial skin compartment (epidermis and HF) and shows dynamic patterns during the hair cycle. Expression of Siah1 appears to be linked to keratinocyte differentiation.

Siah2 is detected in mesenchymal skin compartment (dermis, dermal papilla and dermal sheath). Nevertheless, the intensity of its expression is linked to hair follicle cycle. Siah2 positivity is associated with dynamic changes in dermis during hair growth and degradation. Siah2 also seems to play a role in dermal papilla structural transformations during hair follicle cycle. So, its activity may be associated with papilla functions in hair growth

Therefore, both Siah genes show dynamic patterns of expression during postnatal skin development. These patterns imply their potential role in modulation of hair follicle-specific regulatory pathways (including HIF) in a context-specific manner.

\section{Reference:}

Wenty, Jennifer Lecardonnel, Sandrine Chabane, Annabelle Cousson, Jean Franc, ois Michelet and Bruno A. Bernard "Human Hair Follicle Stem/Progenitor Cells Express Hypoxia Markers" Journal of Investigative Dermatology 2013, 133:20942) Jianfei Qi, Hyungsoo Kim, Marzia Scortegagna, and Ze'ev A Ronai "Regulators and effectors of Siah ubiquitin ligases" Cell Biochemistry And Biophysics $2013,67(1): 15-24$

guide for the recognition and Van Der Veen C, Maurer M, Eichmüller S, Ling G, Hofmann U, Foitzik K, Mecklenburg L, Handjiski B. "A comprehensive 32. 4) Munler-Róver S, Handjski B, van der Veen C, Eichmüller S, Foitzik K, McKay IA, Stenn KS, Paus R. "A comprehensive 